\title{
Assessing the readiness of hospitals in Riyadh Province for efficient and timely stroke management: A pilot study
}

\author{
Zohair A. AlAseri, MD, FRCP EM \& CCM, Fahmi M. Al-Senani, MBBS, MHA, MSc, Shaik S. Ahmed, PhD, PGD, Amena F. Almubarak, \\ MBBS, Dina A. Alzahrani, MBBS, Fatimah A. A. Alzaher, MBBS, Maha A. Altuwaijri, MBBS, Mayar A. Alsudais, MBBS, \\ Rawan Z. Mahgoub, MBBS, Othman Solaiman, MD.
}

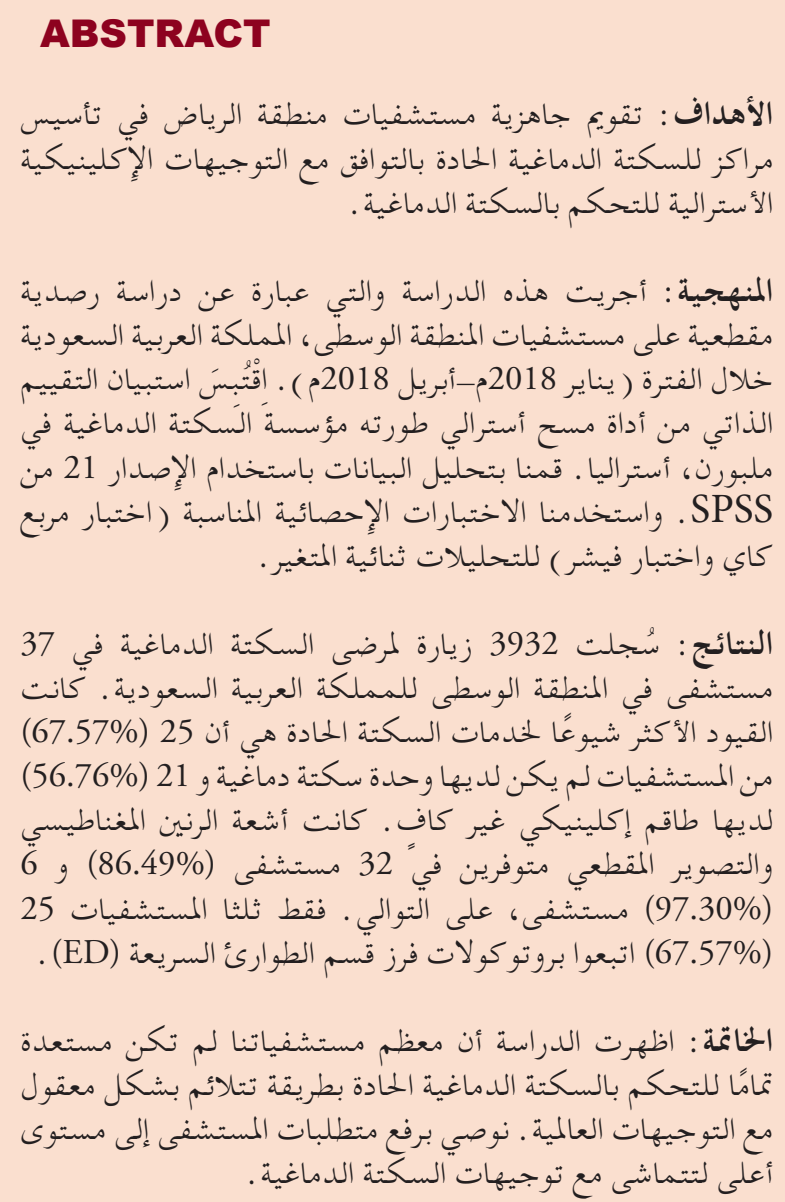

Objectives: To assess the readiness of hospitals in Riyadh to establish acute stroke centers by following the Australian Clinical Guidelines for Stroke Management.

Methods: This study was a quantitative cross-sectional observational study conducted among hospitals in the central region of Saudi Arabia (Jan 2018 - April 2018). A self-administered questionnaire/survey tool was adapted from an Australian survey developed by the Stroke Foundation in Melbourne, Australia.
The data were analyzed using SPSS version 21.0. Appropriate statistical tests (chi-square and Fisher's exact test) were used for bivariate analyses.

Results: A total of 3932 stroke patient visits were recorded in 37 hospitals in the central region of Saudi Arabia. The most common limitations of acute stroke services were that $25(67.57 \%)$ of the hospitals had no stroke unit and $21(56.76 \%)$ had inadequate clinical staff. Magnetic resonance imaging and computed tomography were available in $32(86.49 \%)$ and 36 $(97.30 \%)$ hospitals, respectively. Only two-thirds of hospitals 25 (67.57\%) followed protocols for rapid Emergency Department (ED) triage.

Conclusion: We found that most of our hospitals were not fully prepared to address acute stroke management in a manner that was reasonably consistent with international guidelines. We recommend raising the hospital's requirements a higher level to be in line with the stroke guidelines.

Neurosciences 2021; Vol. 26 (3): 254-260 doi: 10.17712/nsj.2021.3.20200157

From the Departments of Emergency and Critical Care Medicine (Al Aseri), Department of Family and Community (Ahmed), College of Medicine, King Saud University, from the Department of Clinical Sciences (Al Aseri, Almubarak, Alzahrani, Alzaher, Altuwaijri, Mahgoub) Alsudais College of Medicine, Dar Al Uloom University, from the Department of Neurology (Al-Senani), National Neuroscience Institute, and from the Department of Critical Care (Solaiman), King Faisal Specialist Hospital and Research Center, Riyadh, Kingdom of Saudi Arabia.

Received 30th October 2020. Accepted 1st March 2021.

Address correspondence and reprint request to: Dr. Zohair A. Al Aseri, Departments of Emergency and Critical Care Medicine, College of Medicine, King Saud University, Medical City, Riyadh, Kingdom of

Saudi Arabia.E-mail: zohairalaseri@yahoo.com

ORCID ID: https://orcid.org/0000-0001-9869-7544

troke is the second leading cause of death and the
third leading cause of disability worldwide. ${ }^{1}$ The incidence rate of stroke in the Middle East ranges

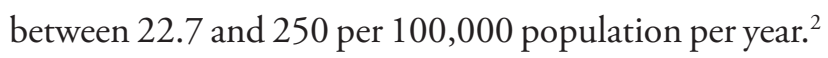


As the young Saudi population ages, the incidence rate of stroke is expected to increase by $57-67 \%$ over the next ten years. ${ }^{3,4}$ Patients with acute ischemic stroke should be evaluated, treated immediately, and approached as having a life-threatening emergency. ${ }^{5}$ The main goal of the clinical guidelines is to enhance the quality of care provided for patients with stroke. They involve aspects of stroke management across the continuum of care. ${ }^{6}$ Data show that timely administration of intravenous tissue-type plasminogen activator (IV tPA) after stroke improves patient outcomes. ${ }^{7}$ The availability of primary stroke centers, stroke units, and comprehensive stroke centers have been demonstrated to provide greater capacity to respond with intravenous thrombolysis as opposed to general hospital treatments. ${ }^{8}$ A modeling study of stroke in Saudi Arabia showed that the development of an up-to-date comprehensive stroke pathway was associated with an increase in clinical functionality, a decrease in disabling strokes and a cost savings of $\$ 602$ million over 15 years in a 10 -year cohort study. ${ }^{9}$ Approaches to improving stroke management should be conducted through a multidisciplinary system to optimize evidence-based acute stroke management delivery. The system should have a well-functioning synchronized unit comprised of neurologists, emergency physicians, nurses, laboratory technicians, radiologists, intervention neuroradiologists, radiology technicians, and admission officers with emergency medical service involvement to pre notify and activate the stroke team in the appropriate situations. ${ }^{10}$ There is no evidence indicating the extent to which evidencebased interventions are provided to acute stroke patients in Saudi Arabian hospitals. Addressing this matter has the potential to identify gaps in current health care services, which could standardize and improve acute stroke management and outcomes for patients with acute stroke. Therefore, this study aimed to assess the readiness of hospitals in Riyadh to establish acute stroke centers that align with the Australian Clinical Guidelines for Stroke Management. ${ }^{11}$ We chose the Australian Clinical Guidelines for Stroke Management as it had already been adapted in a previous study. ${ }^{6}$

Methods. Study design. We searched the MEDLINE, EMBASE, CINAHL, Web of Science, and Cochrane databases in all languages using keywords for the topics

Disclosure. Authors have no conflict of interests, and the work was not supported or funded by any drug company. addressed by the clinical questions, including stroke, readiness, management, assessment, Saudi Arabia, Riyadh, and central region. We could not find any similar study looking at the readiness of Saudi hospitals to provide acute stroke management.

We decided to carry out a quantitative crosssectional observational study among government and private hospitals in the central region of Saudi Arabia (Jan 2018 - April 2018). Data were collected using an Australian survey tool developed by the Stroke Foundation in Melbourne, Australia., ${ }^{11}$ and adapted in Ghana. ${ }^{6}$ Respondents were given the surveys and were met by our team to ensure their understanding of our questions and to maintain quality measures.

Prior permission was obtained from the Saudi Ministry of Health after explaining the objectives of this study. All information on the included hospitals was kept confidential, and data were used for research only. Our data were collected after obtaining ethical approval from the Institutional Review Board (IRB) of King Fahad Medical City (KFMC) and King Saud University (KSU).

Study population. We interviewed 3 respondents per hospital, including medical directors, consultants, head nurses, hospital administrators, neurologists, emergency physicians, chief medical officers (CMOs), and internists in more than 40 hospitals. Our goal was to survey all hospitals with more than 100 beds in Riyadh.

Inclusion and exclusion. All physicians and clinical administrators involved in management of all types of stroke in Riyadh hospitals with bed capacity equal to or greater than 100 beds were included in the study. However, hospitals with a less than 100 beds capacity were excluded from the study.

Statistical analysis. Statistical package for Social Sciences version 21.0 (IBM Inc., Chicago, USA) was used to analyze the data. Descriptive statistics were used to describe all categorical variables. Pearson's chi-square test and Fisher's exact test were used in the bivariate analysis. A $p$-value of $<0.05$ was used to report the statistical significance of the results.

Results. A total of 3932 stroke patient visits in 37 different hospitals in the Central Region of Saudi Arabia were recorded in 2018. Out of 37 different hospitals in the central region of Saudi Arabia, three were teaching hospitals, 2 were military, 13 were private, 8 were referral, and 11 were secondary. Pediatric visits were not recorded as a separate parameter. Referral hospitals represented the majority, accounting for the admittance of $1460(37.1 \%)$ stroke patients in the study year. A total of $28.1 \%$ of stroke patients were admitted to private 
Table 1 - Characteristics of the study hospitals and survey respondents. EP - emergency physicians, CMO - chief medical officer.

\begin{tabular}{|c|c|c|c|}
\hline Hospitals & 2017 stroke admissions & Hospital bed capacity & Survey respondent \\
\hline Teaching hospital 1 & 212 & 200 & Administrator \\
\hline Teaching hospital 2 & 100 & 434 & ICU \\
\hline Teaching hospital 3 & 100 & 607 & Administrator \\
\hline Military hospital 1 & 400 & 1500 & Neurologist \\
\hline Military hospital 2 & 100 & 1679 & Administrator \\
\hline Private hospital 1 & 40 & 354 & $\mathrm{ICU}$ \\
\hline Private hospital 2 & 120 & 300 & Administrator \\
\hline Private hospital 3 & 62 & 405 & EP \\
\hline Private hospital 4 & 48 & 460 & Neurologist \\
\hline Private hospital 5 & 150 & 300 & Neurologist \\
\hline Private hospital 6 & 52 & 250 & $\mathrm{EP}$ \\
\hline Private hospital 7 & 70 & 450 & Administrator \\
\hline Private hospital 8 & 80 & 140 & Administrator \\
\hline Private hospital 9 & 20 & 131 & $\mathrm{CMO}$ \\
\hline Private hospital 10 & 60 & 165 & $\mathrm{CMO}$ \\
\hline Private hospital 11 & 66 & 430 & Administrator \\
\hline Private hospital 12 & 300 & 600 & Administrator \\
\hline Private hospital 13 & 40 & 150 & Administrator \\
\hline Referral hospital 1 & 73 & 200 & Internist \\
\hline Referral hospital 2 & 100 & 500 & Neurologist \\
\hline Referral hospital 3 & 100 & 1200 & Neurologist \\
\hline Referral hospital 4 & 500 & 330 & Neurologist \\
\hline Referral hospital 5 & 200 & 300 & Administrator \\
\hline Referral hospital 6 & 100 & 1500 & Neurologist \\
\hline Referral hospital 7 & 375 & 1200 & Neurologist \\
\hline Referral hospital 8 & 12 & 344 & Administrator \\
\hline Secondary hospital 1 & 16 & 131 & Internist \\
\hline Secondary hospital 2 & 22 & 120 & $\mathrm{CMO}$ \\
\hline Secondary hospital 3 & 20 & 150 & EP \\
\hline Secondary hospital 4 & 35 & 100 & EP \\
\hline Secondary hospital 5 & 100 & 207 & Administrator \\
\hline Secondary hospital 6 & 90 & 178 & Internist \\
\hline Secondary hospital 7 & 40 & 150 & Administrator \\
\hline Secondary hospital 8 & 5 & 164 & Administrator \\
\hline Secondary hospital 9 & 65 & 100 & Administrator \\
\hline Secondary hospital 10 & 9 & 100 & $\mathrm{EP}$ \\
\hline Secondary hospital 11 & 50 & 200 & EP \\
\hline Total & 3932 & & \\
\hline
\end{tabular}

hospitals, 13\% were admitted to military hospitals, and $11.4 \%$ were admitted to secondary hospitals. The lowest admission rate, $10.4 \%$ of patients, was to teaching hospitals. Regarding hospital bed capacity, the military hospital had the highest number of beds among all hospitals. Three referral hospitals also had a larger number of beds than the remaining hospitals, as illustrated in Table 1. Most of the survey respondents were administrators and neurologists (Table 1).
Table 2 illustrates the stroke services and availability in the study hospitals. Approximately two-thirds of our studied hospitals admit patients with stroke symptoms to the ICU in the first 72 hours. Only two-thirds of hospitals followed protocols for rapid ED triage. A total of $54.55 \%$ of secondary hospitals had protocols for the transfer of stroke patients to other hospitals. A total of $72.97 \%$ of hospitals followed clinical management guidelines. Only nine (24.32\%) hospitals have stroke codes. The availability of qualified staff to care for acute 
Table 2 - Stroke service availability in the study hospitals. ICU, intensive care unit; NICE, National Institute for Health and Care Excellence.

\begin{tabular}{|c|c|c|c|c|c|c|}
\hline Stroke services evaluated & $\begin{array}{l}\text { Teaching } \\
\text { hospital }\end{array}$ & $\begin{array}{l}\text { Military } \\
\text { hospital }\end{array}$ & $\begin{array}{c}\text { Private } \\
\text { hospital }\end{array}$ & $\begin{array}{l}\text { Referral } \\
\text { hospital }\end{array}$ & $\begin{array}{c}\text { Secondary } \\
\text { hospital }\end{array}$ & Overall \\
\hline \multicolumn{7}{|c|}{ Which ward is a patient with symptoms most likely to be admitted to for the first $72 \mathrm{~h}$ ? } \\
\hline Acute stroke unit, n (\%) & 0 & $1(50)$ & $1(7.69)$ & $1(12.50)$ & 0 & $3(8.11)$ \\
\hline Emergency department, n (\%) & 0 & $1(50)$ & $1(7.69)$ & 0 & 0 & $2(5.41)$ \\
\hline General medical ward, n (\%) & 0 & 0 & 0 & 0 & $2(18.18)$ & $2(5.41)$ \\
\hline ICU, n (\%) & $2(66.67)$ & 0 & $10(76.92)$ & $3(37.50)$ & $9(81.82)$ & $24(64.86)$ \\
\hline Medical ward, n (\%) & 0 & 0 & $1(7.69)$ & $2(25)$ & 0 & $3(8.11)$ \\
\hline Neurology ward, n (\%) & $1(33.33)$ & 0 & 0 & $2(25)$ & 0 & $3(8.11)$ \\
\hline Protocols for rapid triage, $\mathrm{n}(\%)$ & $2(66.67)$ & $2(100)$ & $8(61.54)$ & $7(87.50)$ & $6(54.55)$ & $25(67.57)$ \\
\hline Arrangements with local ambulances, $\mathrm{n}(\%)$ & $1(33.33)$ & 0 & $5(38.46)$ & $1(12.50)$ & $6(54.55)$ & $13(35.14)$ \\
\hline Protocols for transfer of stroke patients to other hospitals, n (\%) & $1(33.33)$ & 0 & $4(30.77)$ & $2(25)$ & $6(54.55)$ & $13(35.14)$ \\
\hline Clinical management guidelines, $\mathrm{n}(\%)$ & $3(100)$ & $2(100)$ & $9(69.23)$ & $7(87.50)$ & $6(54.55)$ & $27(72.97)$ \\
\hline Standard treatment guidelines, $\mathrm{n}(\%)$ & $1(100)$ & 0 & $3(100)$ & 0 & $1(100)$ & $5(13.51)$ \\
\hline World Stroke Society clinical guidelines, n (\%) & $1(100)$ & 0 & $2(100)$ & 0 & 0 & $3(8.11)$ \\
\hline NICE clinical guidelines, $\mathrm{n}(\%)$ & $1(100)$ & $1(100)$ & $1(100)$ & $1(100)$ & $1(100)$ & $5(13.51)$ \\
\hline American Stroke Association guidelines, n (\%) & 0 & $1(100)$ & $4(100)$ & $2(100)$ & 0 & $7(18.91)$ \\
\hline On-call stroke team, n (\%) & 0 & $2(100)$ & $4(30.77)$ & $5(62.50)$ & 0 & $11(29.73)$ \\
\hline Stroke code, n (\%) & 0 & $2(100)$ & $3(23.08)$ & $4(50)$ & 0 & $9(24.32)$ \\
\hline \multicolumn{7}{|l|}{ 24-h coverage } \\
\hline Emergency consultant, n (\%) & $2(66.67)$ & $2(100)$ & $10(76.92)$ & $7(87.50)$ & $2(18.18)$ & $23(62.16)$ \\
\hline Neuroradiologist, n (\%) & $2(66.67)$ & $2(100)$ & $6(46.15)$ & $7(87.50)$ & $2(18.18)$ & $19(51.35)$ \\
\hline Neurosurgery consultant, $\mathrm{n}(\%)$ & $3(100)$ & $2(100)$ & $13(100)$ & $7(87.50)$ & $2(18.18)$ & $27(72.97)$ \\
\hline Neurologist, n (\%) & $2(66.67)$ & $2(100)$ & $13(100)$ & $8(100)$ & $1(9.09)$ & $26(70.27)$ \\
\hline Stroke physician, n (\%) & $1(33.33)$ & $2(100)$ & $3(23.08)$ & $5(62.50)$ & $1(9.09)$ & $12(32.43)$ \\
\hline ICU consultant, n (\%) & $3(100)$ & $2(100)$ & $13(100)$ & $6(100)$ & $2(18.18)$ & $26(10.27)$ \\
\hline Acute stroke assessment scale, $\mathrm{n}(\%)$ & $2(66.67)$ & $2(100)$ & $9(69.23)$ & $7(87.50)$ & $5(45.45)$ & $25(67.57)$ \\
\hline Dedicated stroke unit, n (\%) & 0 & $1(50)$ & $2(15.38)$ & $2(25)$ & 0 & $5(13.51)$ \\
\hline \multicolumn{7}{|l|}{ Adequate number of beds } \\
\hline Adequate beds, n (\%) & 0 & $1(100)$ & $1(50)$ & $2(100)$ & 0 & $4(10.81)$ \\
\hline More than adequate beds, $\mathrm{n}(\%)$ & 0 & 0 & $1(50)$ & 0 & 0 & $1(2.7)$ \\
\hline \multicolumn{7}{|l|}{ Factors accounting for the lack of stroke unit } \\
\hline Inadequate clinical staff, $\mathrm{n}(\%)$ & $2(100)$ & 0 & $7(100)$ & $4(100)$ & $11(100)$ & $24(64.68)$ \\
\hline Financial constraints, n (\%) & $1(100)$ & 0 & $2(100)$ & $1(100)$ & $1(100)$ & $5(13.51 \%)$ \\
\hline Lack of administrative staff, $\mathrm{n}(\%)$ & $1(100)$ & $1(100)$ & $2(100)$ & 0 & $2(100)$ & $6(16.21 \%)$ \\
\hline Unaware of stroke unit care, n (\%) & 0 & 0 & 0 & 0 & $2(100)$ & $2(5.41)$ \\
\hline Recommended provision of a stroke unit, $\mathrm{n}(\%)$ & $3(100)$ & $2(100)$ & $13(100)$ & $8(100)$ & $8(72.73)$ & $34(91.89)$ \\
\hline Fibrinolytic therapy, n (\%) & $2(66.67)$ & $2(100)$ & $11(84.62)$ & $7(87.50)$ & $5(45.45)$ & $27(72.97)$ \\
\hline Patients received IV Rt-PA, mean \pm std. dev. & $19 \pm 12.72$ & $29.5 \pm 13.4$ & $7.09 \pm 8.57$ & $15.85 \pm 14.75$ & $13 \pm 12.58$ & \\
\hline Inadequate administrative staff, $\mathrm{n}(\%)$ & 0 & 0 & $2(100)$ & 0 & 0 & $2(5.41)$ \\
\hline Surgical treatment for acute stroke, $\mathrm{n}(\%)$ & $2(66.67)$ & $2(100)$ & $11(84.62)$ & $6(75)$ & $1(9.09)$ & $22(59.46)$ \\
\hline Carotid angioplasty, n (\%) & 0 & $2(100)$ & $8(100)$ & $5(100)$ & 0 & $15(40.54)$ \\
\hline Urgent mechanical thrombectomy, n (\%) & $1(100)$ & $2(100)$ & $5(100)$ & $5(100)$ & 0 & $13(35.14)$ \\
\hline Decompressive craniotomy, n (\%) & $2(100)$ & $2(100)$ & $10(100)$ & $6(100)$ & $1(100)$ & $21(56.76)$ \\
\hline Discharge care plans, $\mathrm{n}(\%)$ & $2(66.67)$ & $2(100)$ & $8(61.54)$ & $6(75)$ & $7(63.64)$ & $25(67.57)$ \\
\hline Contact number at discharge, $\mathrm{n}(\%)$ & $1(33.33)$ & $1(50)$ & $9(69.23)$ & $6(75)$ & $4(36.36)$ & $21(56.76)$ \\
\hline
\end{tabular}

stroke patients is illustrated in Table 2. Only 32.43\% of hospitals have 24-h coverage by stroke physicians, and $13.51 \%$ of hospitals had dedicated stroke units. Factors that accounted for the lack of a stroke unit include inadequate clinical staff. A total of $72.97 \%$ of the hospitals used fibrinolytic therapy for acute ischemic stroke, and $56.76 \%$ of hospitals accessed swallow evaluation results within 24 hours. 
Table 2 - Stroke service availability in the study hospitals. ICU, intensive care unit; NICE, National Institute for Health and Care Excellence. (continued)

\begin{tabular}{|c|c|c|c|c|c|c|}
\hline Stroke services evaluated & $\begin{array}{l}\text { Teaching } \\
\text { hospital }\end{array}$ & $\begin{array}{l}\text { Military } \\
\text { hospital }\end{array}$ & $\begin{array}{c}\text { Private } \\
\text { hospital }\end{array}$ & $\begin{array}{l}\text { Referral } \\
\text { hospital }\end{array}$ & $\begin{array}{c}\text { Secondary } \\
\text { hospital }\end{array}$ & Overall \\
\hline \multicolumn{7}{|l|}{ Patient information at admission and discharge } \\
\hline Stroke condition, n (\%) & $1(100)$ & $1(100)$ & $7(100)$ & $4(100)$ & $4(100)$ & $17(45.95)$ \\
\hline Local community care arrangements, $\mathrm{n}(\%)$ & 0 & $1(100)$ & $3(100)$ & 0 & 0 & $4(10.81)$ \\
\hline Local voluntary associations, $\mathrm{n}(\%)$ & 0 & $1(100)$ & 0 & 0 & 0 & $1(2.7)$ \\
\hline Community stroke support groups, n (\%) & 0 & $1(100)$ & 0 & $1(100)$ & 0 & $2(5.41)$ \\
\hline Swallow evaluation within 24 hours, n (\%) & $1(33.33)$ & $2(100)$ & $7(53.85)$ & $7(87.50)$ & $4(36.36)$ & $21(56.76)$ \\
\hline Screening protocol for swallowing, $\mathrm{n}(\%)$ & $1(33.33)$ & $2(100)$ & $6(46.15)$ & $6(75)$ & $2(18.18)$ & $17(45.95)$ \\
\hline \multicolumn{7}{|c|}{ How many of the following professionals are on the stroke team? } \\
\hline Clinical psychologist, (median) & 0.0 & 1.0 & 1.0 & 1.0 & 0.0 & \\
\hline Family medicine, (median) & 0.0 & 8.5 & 1.5 & 0.0 & 0.0 & \\
\hline Neurologist, (median) & 2.0 & 4.0 & 2.0 & 3.5 & 0.0 & \\
\hline Neurosurgeon, (median) & 3.0 & 3.5 & 2.0 & 2.5 & 0.0 & \\
\hline Physician specialist, (median) & 0.0 & 0.5 & 3.0 & 2.0 & 2.0 & \\
\hline Resident, (median) & 1.0 & 3.5 & 1.5 & 2.0 & 5.0 & \\
\hline Stroke care coordinator, (median) & 0.0 & 0.5 & 0.0 & 0.0 & 0.0 & \\
\hline Trained stroke nurse, (median) & 0.0 & 0.5 & 0.0 & 0.0 & 0.0 & \\
\hline Emergency physician, (median) & 3.0 & 12.5 & 4.0 & 7.0 & 4.0 & \\
\hline Occupational therapist, (median) & 0.0 & 3.0 & 0.0 & 0.0 & 0.0 & \\
\hline Physiotherapist, (median) & 2.0 & 1.0 & 5.0 & 3.0 & 1.0 & \\
\hline Speech pathologist, (median) & 0.0 & 1.5 & 1.0 & 2.0 & 0.0 & \\
\hline Social worker, (median) & 1.0 & 1.0 & 2.0 & 2.0 & 1.0 & \\
\hline Dietician, (median) & 2.0 & 1.5 & 2.0 & 1.0 & 1.0 & \\
\hline Specialist for stroke, $\mathrm{n}(\%)$ & $2(66.67)$ & $1(50)$ & $5(38.46)$ & $6(75)$ & $3(27.27)$ & $17(45.95)$ \\
\hline Program for professional development, $\mathrm{n}(\%)$ & 0 & $1(50)$ & $3(23.08)$ & $4(50)$ & $3(27.27)$ & $11(29.73)$ \\
\hline Stroke team involved in quality improvement, $\mathrm{n}(\%)$ & $1(33.33)$ & $1(50)$ & $5(38.46)$ & $5(62.50)$ & $1(9.09)$ & $13(35.14)$ \\
\hline \multicolumn{7}{|l|}{ Health policy support level (national) } \\
\hline High support, n (\%) & 0 & 0 & $2(5.38)$ & 0 & 0 & $2(5.41)$ \\
\hline Average support, $\mathrm{n}(\%)$ & $2(66.67)$ & $2(100)$ & $7(53.85)$ & $3(37.50)$ & $2(18.18)$ & $16(43.24)$ \\
\hline Limited support, n (\%) & 0 & 0 & $2(15.38)$ & $3(37.50)$ & $5(45.45)$ & $10(27.03)$ \\
\hline Low support, n (\%) & 0 & 0 & 0 & 0 & $4(36.36)$ & $4(10.81)$ \\
\hline No support, n (\%) & $1(33.33)$ & 0 & $2(15.38)$ & $2(25)$ & 0 & $5(13.51)$ \\
\hline \multicolumn{7}{|l|}{ Health policy support level (hospital) } \\
\hline High support, n (\%) & $1(33.33)$ & $1(50)$ & $4(30.77)$ & $2(25)$ & 0 & $8(21.62)$ \\
\hline Average support, $\mathrm{n}(\%)$ & $1(33.33)$ & $1(50)$ & $8(61.54)$ & $4(50)$ & $4(36.36)$ & $18(48.65)$ \\
\hline Limited support, $\mathrm{n}(\%)$ & 0 & 0 & $1(7.69)$ & $1(12.50)$ & $2(18.18)$ & $4(10.81)$ \\
\hline Low support, n (\%) & 0 & 0 & 0 & 0 & $4(36.36)$ & $4(10.81)$ \\
\hline No support, n (\%) & $1(33.33)$ & 0 & 0 & $1(12.50)$ & $1(9.09)$ & $3(8.11)$ \\
\hline \multicolumn{7}{|l|}{ Limitations of the acute stroke services } \\
\hline No ward, n (\%) & $2(100)$ & $1(100)$ & $7(100)$ & $5(100)$ & $10(100)$ & $25(67.57)$ \\
\hline Inadequate clinical staff, n (\%) & $1(100)$ & $1(100)$ & $6(100)$ & $4(100)$ & $9(100)$ & $21(56.76)$ \\
\hline Financial constraints, $\mathrm{n}(\%)$ & $1(100)$ & $1(100)$ & $8(100)$ & $3(100)$ & $4(100)$ & $17(45.95)$ \\
\hline Health policy support, $\mathrm{n}(\%)$ & $2(100)$ & $1(100)$ & $7(100)$ & $3(100)$ & $3(100)$ & $16(43.24)$ \\
\hline Lack of political will, $n(\%)$ & $2(100)$ & $1(100)$ & $2(100)$ & $3(100)$ & $2(100)$ & $10(27.03)$ \\
\hline Stroke register or database, $\mathrm{n}(\%)$ & 0 & $2(100)$ & $2(15.38)$ & $3(37.50)$ & $2(18.18)$ & $9(24.32)$ \\
\hline Interventions to promote stroke awareness, $\mathrm{n}(\%)$ & $1(33.33)$ & $2(100)$ & $9(69.23)$ & $5(62.50)$ & $2(18.18)$ & $19(51.35)$ \\
\hline Rehabilitative programs, n (\%) & $2(66.67)$ & $1(50)$ & $3(23.08)$ & $2(25)$ & 0 & $8(21.62)$ \\
\hline
\end{tabular}


Table 3 - World Stroke Organization checklist. MRA, magnetic resonance angiography; EEG, electroencephalogram; MRI, magnetic resonance imaging; CT, computed tomography.

\begin{tabular}{lcccccc}
\hline Component of acute stroke services & $\begin{array}{c}\text { Teaching } \\
\text { hospital }\end{array}$ & $\begin{array}{c}\text { Military } \\
\text { hospital }\end{array}$ & $\begin{array}{c}\text { Private } \\
\text { hospital }\end{array}$ & $\begin{array}{c}\text { Referral } \\
\text { hospital }\end{array}$ & $\begin{array}{c}\text { Secondary } \\
\text { hospital }\end{array}$ & Overall \\
\hline MRA, n (\%) & $3(100)$ & $2(100)$ & $13(100)$ & $7(87.50)$ & $2(18.18)$ & $27(72.97)$ \\
Computed tomographic angiography, n (\%) & $3(100)$ & $2(100)$ & $13(100)$ & $6(75)$ & $1(9.09)$ & $25(67.57)$ \\
EEG services availability, n (\%) & $1(33.33)$ & $2(100)$ & $12(92.31)$ & $7(87.50)$ & $1(9.09)$ & $23(62.16)$ \\
Echocardiogram services, $\mathrm{n}(\%)$ & $2(66.67)$ & $2(100)$ & $13(100)$ & $8(100)$ & $5(45.45)$ & $30(81.08)$ \\
Neurovascular ultrasound, n (\%) & $2(66.67)$ & $2(100)$ & $12(92.31)$ & $8(100)$ & $5(45.45)$ & $29(78.38)$ \\
MRI, n (\%) & $3(100)$ & $2(100)$ & $13(100)$ & $8(100)$ & $6(54.55)$ & $32(86.49)$ \\
CT scanner, n (\%) & $3(100)$ & $2(100)$ & $13(100)$ & $8(100)$ & $10(90.91)$ & $36(97.30)$ \\
Arteriovenous malformation treatment, n (\%) & $1(100)$ & $2(100)$ & $6(100)$ & $5(100)$ & 0 & $14(37.84)$ \\
Surgery for cerebral aneurysm, n (\%) & $1(100)$ & $2(100)$ & $5(100)$ & $5(100)$ & 0 & $13(35.14)$ \\
Carotid endarterectomy, n (\%) & $1(100)$ & $2(100)$ & $4(100)$ & $5(100)$ & 0 & $12(32.43)$ \\
Nurses, mean \pm std. dev. & $2.6 \pm 4.61$ & $8.5 \pm 10.60$ & $7.1 \pm 5.46$ & $9.14 \pm 13.65$ & $5.11 \pm 3.58$ & \\
Aspirin provided for eligible patients, n (\%) & $3(100)$ & $2(100)$ & $11(84.62)$ & $8(100)$ & $11(100)$ & $35(94.59)$ \\
Effective rehabilitation services, $\mathrm{n}(\%)$ & $2(66.67)$ & $2(100)$ & $7(53.85)$ & $4(50)$ & $4(36.36)$ & $19(51.35)$ \\
\hline
\end{tabular}

Regarding the number of professionals on the stroke team, clinical psychologists and family medicine physicians were common in the military, referral, and private hospitals; neurosurgeons, trained stroke nurses, emergency physicians, and speech pathologists were also common among the military hospitals. Neurologists were most common in teaching hospitals. Stroke care coordinators, physiotherapists, social workers, and dieticians were most common in the military and private hospitals. Occupational therapists were the most common specialist in military hospitals. Only $11(29.73 \%)$ hospitals had a program for professional development. A total of $35.14 \%$ of all hospitals had a stroke team involved in quality improvement.

Concerning the health policy support level, $48.65 \%$ of the hospitals had average support, $21.62 \%$ had high support, and the best level of support was observed in the military hospitals.

Regarding the limitations of the acute stroke services, more than two-thirds of hospitals had no wards, $56.76 \%$ had inadequate clinical staff, only $24.32 \%$ had stroke registries or databases, $51.35 \%$ had interventions to promote stroke awareness, and $21.62 \%$ had no rehabilitative programs.

Table 3 illustrates the compliance of the studied hospitals with the World Stroke Organization checklist. Magnetic resonance angiography (MRA) is highly used in most of our hospitals. The same finding was observed for the use of a diagnostic tool, with frequent availability of computed tomographic angiography and electroencephalogram (EEG) services. Echocardiogram services were used in $62.16 \%$ of all hospitals.
Discussion. This study highlights critical points regarding acute stroke management in the central region of the Kingdom of Saudi Arabia. Our study reported a total of 3932 stroke patient visits to 37 different hospitals in the central region of Saudi Arabia in 2018. Only two-thirds of these hospitals followed protocols for rapid ED triage.

Understanding the enormous impact of time on a patient's outcome should promote changes and modifications in stroke management to decrease delays. The American Stroke Association identified strategies associated with reduction in the door-to-IV tPA time in stroke patients. ${ }^{12}$ Early notification of emergency medical services, emergency department recognition of stroke, and the triage system play essential roles in decreasing the door-to-needle time in acute stroke management. ${ }^{13,14}$

After transient ischemic attack or minor stroke, the risk of consequent stroke is lower for patients treated by services that provide emergency intervention using a multidisciplinary approach. ${ }^{15}$ Our results showed that in only approximately two-thirds of our studied hospitals, patients with stroke symptoms were most likely to be admitted to the ICU in the first $72 \mathrm{~h}$, only $35.14 \%$ of hospitals had arrangements with a local ambulance.

We reported many factors that account for the lack of stroke units, including some hospitals having an inadequate number of trained clinical staff and a lack of administrative support, among other factors. In our study, the primary diagnostic tools, including magnetic resonance angiography (MRA), electroencephalogram (EEG), magnetic resonance imaging (MRI), computed 
tomography CT, and echocardiogram, were available in most of the hospitals. Only half of our hospitals had effective rehabilitation services.

Our study lays the groundwork to start implementing stroke guidelines and create well-organized stroke units in Riyadh and, hopefully, in other hospitals around the Kingdom, which will impact the health care system in accordance with the Kingdom's 2030 vision. We recommend enhancing acute stroke management at all health care layers including hospital resources, infrastructures, rapid admission of stroke patient to qualified hospital, emergency transport and triage. Further research is needed to make sure that our hospitals align with Saudi stroke management guidelines.

Limitations. This study was conducting using a questionnaire that had been previously validated in Australia and Ghana. A limitation of this study, similar to many questionnaire studies, is its subjectivity and recall bias.

Conclusion. We found that most of our hospitals were not fully prepared to address acute stroke management in a manner that was reasonably consistent with international guidelines. We recommend raising the hospital's requirements a higher level to be in line with the stroke guidelines.

Acknowledgements. The authors would like to thank American Journal Experts (www.aje.com) for English language editing.

\section{References}

1. World Health Organization. The top 10 causes of death. 9 December 2020. https://www.who.int/news-room/fact-sheets/ detail/the-top-10-causes-of-death

2. El-Hajj M, Salameh P, Rachidi S, Hosseini H. The epidemiology of stroke in the Middle East. Eur Stroke J 2016; 1: 180-198.

3. Jan MM, Qari FA. Epidemiology of stroke in Saudi Arabia. Saudi Med J 2001; 22: 375-376.

4. Al-Senani F, Al-Johani M, Salawati M, Alhazzani A, Morgenstern LB, Seguel Ravest V, et al. An epidemiological model for first stroke in Saudi Arabia. J Stroke Cerebrovasc Dis 2020; 29: 104465.
5. Powers WJ, Rabinstein AA, Ackerson T, Adeoye OM, Bambakidis NC, Becker K, et al. 2018 guidelines for the early management of patients with acute ischemic stroke: a guideline for healthcare professionals from the American Heart Association/American Stroke Association. Stroke 2018; 49: e46-e110.

6. Baatiema L, Otim M, Mnatzaganian G, Aikins AD, Coombes J, Somerset S. Towards best practice in acute stroke care in Ghana: a survey of hospital services. BMC Health Serv Res 2017; 17: 108.

7. Kim JT, Fonarow GC, Smith EE, Reeves MJ, Navalkele DD, Grotta JC, et al. Treatment with tissue plasminogen activator in the golden hour and the shape of the 4.5-hour time-benefit curve in the national United States get with the guidelinesstroke population. Circulation 2017; 135: 128-139.

8. Masjuan J, Gállego Culleré J, Ignacio García E, Mira Solves JJ, Ollero Ortiz A, Vidal de Francisco D, et al. Stroke treatment outcomes in hospitals with and without Stroke Units. Neurologia 2020; 35: 16-23.

9. Al-Senani F, Al-Johani M, Salawati M, ElSheikh S, AlQahtani M, Muthana J, et al. A national economic and clinical model for ischemic stroke care development in Saudi Arabia: a call for change. Int J Stroke 2019; 14: 835-842.

10. Jeon SB, Ryoo SM, Lee DH, Kwon SU, Jang S, Lee EJ, et al. Multidisciplinary approach to decrease in-hospital delay for stroke thrombolysis. J Stroke 2017; 19: 196-204.

11. Stroke Foundation. Clinical guidelines for stroke management. Melbourne Australia, 2017. Available from: https:// informme.org.au/Guidelines/Clinical-Guidelines-for-StrokeManagement. Accessed October 30, 2020

12. Xian Y, Xu H, Lytle B, Blevins J, Peterson ED, Hernandez AF, et al. Use of strategies to improve door-to-needle times with tissue-type plasminogen activator in acute ischemic stroke in clinical practice: findings from target: Stroke. Circ Cardiovasc Qual Outcomes 2017; 10: e003227.

13. Hsieh MJ, Tang SC, Chiang WC, Tsai LK, Jeng JS, Ma $\mathrm{MH}$. Effect of prehospital notification on acute stroke care: a multicenter study. Scand J Trauma Resusc Emerg Med 2016; 24: 57.

14. Miura S, Miyata R, Matsumoto S, Higashi T, Wakisaka Y, Ago T, et al. Quality management program of stroke rehabilitation using adherence to guidelines: a nationwide initiative in Japan. J Stroke Cerebrovasc Dis 2019; 28: 2434-2441.

15. Hartford W, Lear S, Nimmon L. Stroke survivors' experiences of team support along their recovery continuum. BMC Health Serv Res 2019; 19: 723. 